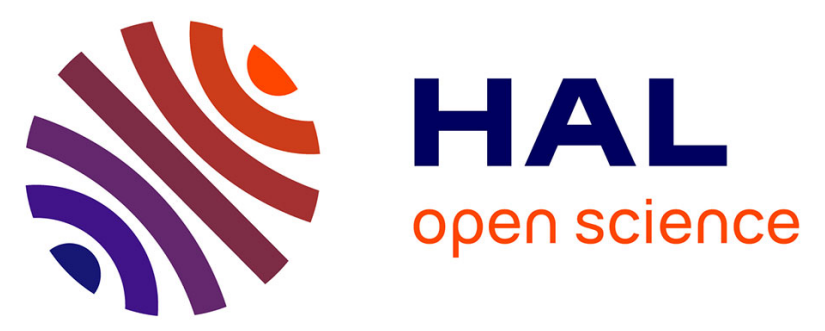

\title{
Paradoxical effects of endurance training and chronic hypoxia on myofibrillar ATPase activity.
}

\author{
Belle Roels, Carlo Reggiani, Cyril Reboul, Corinne Lionne, B. Iorga, Philippe
} Obert, Stéphane Tanguy, A. Gibault, A. Jougla, F. Travers, et al.

\section{- To cite this version:}

Belle Roels, Carlo Reggiani, Cyril Reboul, Corinne Lionne, B. Iorga, et al.. Paradoxical effects of endurance training and chronic hypoxia on myofibrillar ATPase activity.. AJP - Regulatory, Integrative and Comparative Physiology, 2008, 294 (6), pp.R1911-8. 10.1152/ajpregu.00210.2006 . hal00311705

\section{HAL Id: hal-00311705 \\ https://hal.science/hal-00311705}

Submitted on 31 May 2020

HAL is a multi-disciplinary open access archive for the deposit and dissemination of scientific research documents, whether they are published or not. The documents may come from teaching and research institutions in France or abroad, or from public or private research centers.
L'archive ouverte pluridisciplinaire HAL, est destinée au dépôt et à la diffusion de documents scientifiques de niveau recherche, publiés ou non, émanant des établissements d'enseignement et de recherche français ou étrangers, des laboratoires publics ou privés. 


\section{B. Roels, C. Reggiani, C. Reboul, C. Lionne, B. Iorga, P. Obert, S. Tanguy, A.}

Gibault, A. Jougla, F. Travers, G. P. Millet and R. Candau

Am J Physiol Regulatory Integrative Comp Physiol 294:1911-1918, 2008. First published Apr 16, 2008; doi:10.1152/ajpregu.00210.2006

You might find this additional information useful...

This article cites 31 articles, 13 of which you can access free at:

http://ajpregu.physiology.org/cgi/content/full/294/6/R1911\#BIBL

Updated information and services including high-resolution figures, can be found at:

http://ajpregu.physiology.org/cgi/content/full/294/6/R1911

Additional material and information about American Journal of Physiology - Regulatory, Integrative and Comparative Physiology can be found at:

http://www.the-aps.org/publications/ajpregu

This information is current as of September 7, 2010 .

The American Journal of Physiology - Regulatory, Integrative and Comparative Physiology publishes original investigations that illuminate normal or abnormal regulation and integration of physiological mechanisms at all levels of biological organization, ranging from molecules to humans, including clinical investigations. It is published 12 times a year (monthly) by the American

Physiological Society, 9650 Rockville Pike, Bethesda MD 20814-3991. Copyright @ 2008 by the American Physiological Society. ISSN: 0363-6119, ESSN: 1522-1490. Visit our website at http://www.the-aps.org/. 


\title{
Paradoxical effects of endurance training and chronic hypoxia on myofibrillar
}

\section{ATPase activity}

\author{
B. Roels, ${ }^{1,2,3}$ C. Reggiani, ${ }^{2}$ C. Reboul,${ }^{4}$ C. Lionne, ${ }^{5}$ B. Iorga, ${ }^{5,6}$ P. Obert, ${ }^{4}$ S. Tanguy,${ }^{4}$ A. Gibault, ${ }^{4}$ \\ A. Jougla, ${ }^{1}$ F. Travers, ${ }^{5}$ G. P. Millet, ${ }^{1}$ and R. Candau ${ }^{1,5}$ \\ ${ }^{1}$ UMR 866 Institut National de la Recherche Agronomique, University of Montpellier I, Faculty of Sport Sciences, \\ Montpellier, France; ${ }^{2}$ Department of Anatomy and Physiology, University of Padova, Padova, Italy; ${ }^{3}$ Brunel University, \\ School of Sport and Education, West-London, UK; ${ }^{4}$ EA 2426 Laboratory of Cardiovascular Adaptations to Exercise, Avignon, \\ France; ${ }^{5}$ UMR 5236 Centre National de la Recherche Scientifique, University of Montpellier I, France; \\ and ${ }^{6}$ Department of Physics and Applied Mathematics, Faculty of Chemistry, University of Bucharest, Bucharest, Romania
}

Submitted 24 March 2006; accepted in final form 26 March 2008

Roels B, Reggiani C, Reboul C, Lionne C, Iorga B, Obert P, Tanguy S, Gibault A, Jougla A, Travers F, Millet GP, Candau R. Paradoxical effects of endurance training and chronic hypoxia on myofibrillar ATPase activity. Am J Physiol Regul Integr Comp Physiol 294: R1911-R1918, 2008. First published April 16, 2008; doi:10.1152/ajpregu.00210.2006.-This study aimed to determine the changes in soleus myofibrillar ATPase (m-ATPase) activity and myosin heavy chain (MHC) isoform expression after endurance training and/or chronic hypoxic exposure. Dark Agouti rats were randomly divided into four groups: control, normoxic sedentary $(\mathrm{N} ; n=14)$, normoxic endurance trained (NT; $n=14)$, hypoxic sedentary $(\mathrm{H} ; n=10)$, and hypoxic endurance trained (HT; $n=14)$. Rats lived and trained in normoxia at $760 \mathrm{mmHg}$ (N and NT) or hypobaric hypoxia at $550 \mathrm{mmHg}(\sim 2,800 \mathrm{~m})$ (H and HT). m-ATPase activity was measured by rapid flow quench technique; myosin subunits were analyzed with mono- and two-dimensional gel electrophoresis. Endurance training significantly increased m-ATPase $(P<0.01)$, although an increase in MHC-I content occurred $(P<0.01)$. In spite of slow-to-fast transitions in MHC isoform distribution in chronic hypoxia $(P<0.05)$ no increase in m-ATPase was observed. The rate constants of $\mathrm{m}$-ATPase were $0.0350 \pm 0.0023 \mathrm{~s}^{-1}$ and $0.047 \pm 0.0050 \mathrm{~s}^{-1}$ for N and NT and $0.033 \pm 0.0021 \mathrm{~s}^{-1}$ and $0.038 \pm 0.0032 \mathrm{~s}^{-1}$ for $\mathrm{H}$ and HT. Thus, dissociation between variations in m-ATPase and changes in MHC isoform expression was observed. Changes in fraction of active myosin heads, in myosin light chain isoform (MLC) distribution or in MLC phosphorylation, could not explain the variations in m-ATPase. Myosin posttranslational modifications or changes in other myofibrillar proteins may therefore be responsible for the observed variations in m-ATPase activity.

myosin ATPase activity; myosin heavy chain isoform; myosin light; chain isoform; exercise; fast kinetics

CHRONIC HYPOXIC EXPOSURE INDUCES several structural modifications in skeletal muscles, that is, a decrease in fiber crosssectional area and in muscle mass, mainly due to an anorexic effect. Even when the anorexic effect is controlled by using a pair-fed experimental design, the composition of myosin heavy chain (MHC) is changed, and an increase in the fast-type isoforms is observed. Indeed, a shift toward glycolytic-oxidative fibers (MHC-IIA) in muscles of rats exposed to hypoxia is generally reported, except in the study of Sillau and Banchero (35), in which no difference was shown in the fiber type composition of rat soleus after hypoxic exposure. In addition, Bigard et al. (7) showed that $4 \mathrm{wk}$ in hypobaric hypoxia resulted in a decrease in MHC-I in the soleus of young rats.

Address for reprint requests and other correspondence: B. Roels, Orion Clinical Services, 7 Bath Rd., Slough, Berkshire SL1 3UE UK (e-mail: belleroels@hotmail.com).
Thus, hypoxia seems to affect the structural and biochemical properties of skeletal muscles by inducing a transformation from type I to type II fibers.

Opposite to chronic hypoxia, endurance training is likely to induce a decrease in fast fibers and an increase in slow-fiber proportion. For instance, Green et al. (18) demonstrated that a prolonged (15 wk) endurance training induced a fiber transformation from fast-type IIa to slow type I on the basis of enzyme histochemistry and MLC distribution. Endurance training (10 wk) also induced changes in the distribution patterns of fast alkali MLC isoforms detectable within FMb (triplet of isomyosins with distinct MLC complement, FM1b, FM2b, and FM3b) composed of the fastest isoform MHC-IIB (3). Surprisingly, endurance training seems to induce an increase in the actomyosin ATPase activity and maximal shortening velocity in the slow-twitch soleus muscle and a decrease in the fast region of the vastus lateralis (34). These observations of changes in ATPase activity and myosin isoform shifts appear to be paradoxical, as ATP hydrolyzed by myosin represents the main component of ATP consumption during contraction. These changes in myosin isoform expression probably regulate ATP consumption. Actually, there is strong evidence that myosin isoforms are the main determinant of ATPase activity, at least at low temperature in vitro. In this study, we analyzed myosin isoform composition and myofibrillar ATPase activity in soleus muscles of rats exposed to normoxia or hypoxia with or without endurance training to assess the variations of soleus myofibrillar ATPase (m-ATPase) activity that accompany the changes in MHC isoform expression under training and/or chronic hypoxia conditions.

\section{METHODS}

Animal model. Sixteen-week-old, sea level native dark Agouti male rats $(260 \pm 23 \mathrm{~g})$, obtained from Harlan Laboratories (Gannat, Puy de Dôme, France), were randomly assigned to one of the following four groups: hypobaric hypoxia with endurance training sessions (HT rats, $n=14$ ), hypobaric hypoxia without training ( $\mathrm{H}$ rats, $n=10)$, normoxia with endurance training sessions (NT rats, $n=14$ ), or normoxia without training ( $\mathrm{N}$ rats, $n=14$ ).

Hypoxic exposure protocol. Hypobaric hypoxia was obtained in steel chambers [fitted with a clear plastic glass door to illuminate and observe the animals] by using a specific vacuum pump (Becker Mot63, Rambouillet, France) as previously described by Melin et al.

The costs of publication of this article were defrayed in part by the payment of page charges. The article must therefore be hereby marked "advertisement" in accordance with 18 U.S.C. Section 1734 solely to indicate this fact. 
(28). In each chamber, barometric pressure (MPX2100AP, Conrad, Lille, France), humidity (GSH 14; Conrad), and temperature (PT100/ DINB; Conrad) conditions were continuously monitored by using electronic sensors and analyzed with Direct View software (5500MF; ADAC, Atlanta, GA). All rats were maintained for $5 \mathrm{wk}$ in their own environment, at a barometric pressure of $760 \mathrm{mmHg}\left(\mathrm{PI}_{\mathrm{O}_{2}} \sim 159 \mathrm{mmHg}\right.$, altitude $\sim 80 \mathrm{~m}$ ) for $\mathrm{N}$ and $\mathrm{NT}$, or $550 \mathrm{mmHg}\left(\mathrm{PI}_{\mathrm{O}_{2}} \sim 105 \mathrm{mmHg}\right.$, altitude $\sim 2,800 \mathrm{~m}$ ) for $\mathrm{H}$ and HT. The level and duration of the hypoxic stimulus were suggested to be sufficient for inducing adaptations, while still well tolerated by the rats and without any negative effects of the hypoxic stimulus, such as muscle mass loss, sickness, or fatigue, compared with controls (10). H and HT animals were fed ad libitum with free access to tap water. Because of the hypoxic effect on food intake and consequently animal growth, a pair-fed regimen was applied to the two other groups, with free access to tap water. To assure there was no decrease in body weight due to the hypoxic exposure, the rats' body weight was measured before and after the protocol. Room temperature was maintained at $\sim 21^{\circ} \mathrm{C}$ using air-conditioning. Not more than four animals were kept at the same time in each cage. A 12:12-h light-dark cycle was applied. All procedures were performed in agreement with the Guide for the Care and Use of Laboratory Animals published by the U.S. National Institutes of Health (NIH Publications No. 85-23, revised 1996) and with the approval of the French Ministry of Agriculture.

Training program. Twenty-five training sessions were conducted in NT and HT rats during the $5 \mathrm{wk}$ of environmental exposure; that is, five sessions a week followed by two consecutive days of rest at the end of the week. Training consisted in wheel-driven running (EA 2426, Avignon, France) at $80 \%$ of maximal aerobic velocity (MAV); that is, the maximal speed reached at the end of the incremental test described below. To define accurately the training intensities, MAV was evaluated before the study period in normoxia or hypoxia. MAVs were also measured during the third week to adjust the training intensities. MAVs were evaluated using a driven wheel during a continuous incremental exercise test to exhaustion. Under normoxia, the driven wheel was set at a speed of $10 \mathrm{~m} / \mathrm{min}$ for 2 to $3 \mathrm{~min}$, after which the speed was increased by $4 \mathrm{~m} / \mathrm{min}$ every $90 \mathrm{~s}$ until $85-90 \%$ of the expected MAV was reached. Then the speed was increased by $0.5-1 \mathrm{~m} / \mathrm{min}$ every $60 \mathrm{~s}$ until MAV was reached. Hypoxic MAV was evaluated using the same protocol, but with a starting speed of 7 $\mathrm{m} / \mathrm{min}$. In both cases, we considered that MAV was reached when the rat was not able to follow the speed imposed by the driven wheel anymore and turned into the wheel more than three times. MAV reproducibility was evaluated during a preliminary study in five dark Agouti rats submitted to three MAV tests, each test being performed with a 2-day interval. Individual variation coefficients were lower than $4.6 \%$, suggesting a good reliability of the measurements. Training lasted $5 \mathrm{wk}$ and was conducted at the same relative intensity for both groups (i.e., $80 \%$ of normoxic MAV for NT and $80 \%$ of hypoxic MAV for HT). Each session lasted about $20 \mathrm{~min}$ in the first week and was increased up to $60 \mathrm{~min}$ in the last week. Rats were trained at the same $\mathrm{PI}_{\mathrm{O}_{2}}$ experienced for the rest of the day. The running skills of the $\mathrm{N}$ and $\mathrm{H}$ group were maintained by performing two sessions a week of $10 \mathrm{~min}$ at $40 \%$ of MAV. This training program was chosen, as it has been used in our laboratory for several years and is effective to induce changes in MAV, muscular, and cardiovascular parameters $(16,28,31)$.

Myofibril preparation. At the end of the 5-wk training protocol, all of the rats were anesthetized with intraperitoneal ketamine and xylazine injection ( $50 \mathrm{mg} / \mathrm{kg}$ body mass), and killed. Both soleus muscles were quickly removed. In each animal, one soleus was immediately frozen in liquid nitrogen $\left(\mathrm{N}_{2}\right)$ for electrophoretic analysis, and one soleus was immersed in ice-cooled Ringer buffer $(50 \mathrm{mM}$ Tris $\cdot \mathrm{HCl}$, pH 7.0, $100 \mathrm{mM} \mathrm{NaCl}, 2 \mathrm{mM} \mathrm{KCl}, 2 \mathrm{mM} \mathrm{MgCl} 2,1 \mathrm{mM}$ EGTA, 2 mM DTT, $0.2 \mathrm{mM}$ PMSF, $10 \mu \mathrm{M}$ leupeptin, $5 \mu \mathrm{M}$ pepstatin, and 0.5 $\mathrm{mM} \mathrm{NaN}_{3}$ ) at slack length. From these latter soleus muscles, myofi- brils were prepared as previously described (11). The total myosin head concentration in the myofibrillar suspension was measured by absorption at $280 \mathrm{~nm}$ of a $1 / 20$ dilution of the suspension in $2 \%$ SDS (23). Sarcomere length of the myofibrils was measured as previously described (11). Ten to twenty myofibrils were analyzed, and their sarcomere lengths were averaged.

Myofibrillar ATPase activity. ATPase measurements were carried out in a home-built, thermostatically controlled, rapid flow quench apparatus (4). Experiments were performed at $4{ }^{\circ} \mathrm{C}$ to slow down the unloaded shortening velocity (V0), and thus, to increase the duration of the unloaded shortening phase $\left(t_{\mathrm{B}}\right)$. The chosen temperature of $4^{\circ} \mathrm{C}$, although slowing the shortening velocity down, still allows the myofibrils to behave and contract normally and offers the possibility to measure the rate constant of the cleavage step in the cross bridge cycle, as validated by Stehle et al. (36). In the rapid flow quench apparatus, myofibrils were mixed with $\left[\gamma^{-32} \mathrm{P}\right] \mathrm{ATP}(2.5 \mu \mathrm{M}$ myosin heads $+25 \mu \mathrm{M}[\gamma-32 \mathrm{P}]$ ATP in reaction mixture at $4^{\circ} \mathrm{C}$ in $\mathrm{Ca}^{2+}$ activated condition). Reaction mixtures were quenched in acid (22\% TCA and $1 \mathrm{mM} \mathrm{KH}_{2} \mathrm{PO}_{4}$ ) at different times, and the $\left[{ }^{32} \mathrm{P}\right] \mathrm{Pi}$ produced was assayed. Reaction mixtures with incubation times from $200 \mathrm{~ms}$ to tens of seconds were obtained with this apparatus. For longer incubation times, myofibrils and $\left[\gamma-{ }^{32} \mathrm{P}\right]$ ATP were mixed in a thermostated beaker, where one sample of the reaction mixture was taken and quenched every $10-15$ s. The amounts of total Pi (i.e., free Pi plus myosin head bound $\mathrm{Pi}$ ) were determined in the quenched reaction mixtures by the filter paper method of Reimann and Umfleet (32). Relaxing and activating buffers were used to obtain relaxed and $\mathrm{Ca}^{2+}$-activated ATPase rates, respectively. Buffers consisted of 50 $\mathrm{mM}$ Tris-acetate, $\mathrm{pH} 7.4,100 \mathrm{mM} \mathrm{K}$ acetate, $5 \mathrm{mM} \mathrm{KCl}$, and either $0.1 \mathrm{mM} \mathrm{CaCl}_{2}, 2 \mathrm{mM} \mathrm{Mg}$-acetate for the activating buffer $\left(+\mathrm{Ca}^{2+}\right)$ or $2 \mathrm{mM} \mathrm{EGTA}$, and $5 \mathrm{mM} \mathrm{Mg}$-acetate for the relaxing buffer (without $\mathrm{Ca}^{2+}$ ). Experiments were carried out in paired conditions to control external factors. In the same day, the steady-state rates of myosin ATPase activity were measured in training vs. control group or in hypoxia $v s$. control group with precisely the same procedure of myofibril preparation, the same buffers, and temperature. Three independent sets of experiments on myofibrillar ATPase parameters, with different myofibrils samples, were carried out to ensure intertrial consistency.

Modeling of the ATPase activity kinetics. The first phase of steadystate ATP hydrolysis corresponds to the ATPase activity of $\mathrm{Ca}^{2+}$. activated myofibrils during unloaded shortening. The second phase that is slower was shown to represent the ATPase activity of overcontracting myofibrils (26). Overcontraction starts when the thin filaments of the two halves of the sarcomeres start to overlap with each other. The whole kinetics of ATP hydrolysis was modeled with a three-component model, one exponential (transient Pi burst) and two linear (steady states): [Total Pi]/[myosin head $]=\mathrm{A}\left(1-\mathrm{e}^{- \text {kobs } \cdot t}\right)+$ $k_{\mathrm{F}} \cdot t+U \cdot\left[\mathrm{A}+k_{\mathrm{F}} \cdot t_{\mathrm{B}}+\mathrm{k}_{\mathrm{S}}\left(t-t_{\mathrm{B}}\right)\right]$, where [Total Pi]/[myosin head] is the amount of total phosphate determined per myosin head (mol of ATP hydrolyzed $/ \mathrm{mol}$ of myosin head), A and $k_{\text {obs }}$ are the amplitude $(\mathrm{mol} / \mathrm{mol})$ and the rate constant $\left(\mathrm{s}^{-1}\right)$ of the Pi burst respectively, $k_{\mathrm{F}}$ is the rate constant $\left(\mathrm{s}^{-1}\right)$ of the steady state during unloading shortening phase, $k_{\mathrm{S}}$ is the rate constant $\left(\mathrm{s}^{-1}\right)$ of the steady state during overcontraction, $t_{\mathrm{B}}$ is the time break (s) corresponding to the unloading shortening phase duration, $U=0$, when $t<t_{\mathrm{B}}$ and $U=1$, when $t>t_{\mathrm{B}}$. The third component starts at the offset of the first fast linear phase. The model parameters were determined using an iterative process that minimizes the sum of the mean squares between the total phosphate measured and that predicted by the model.

Titration of active myosin heads. In Pi burst experiments, the titration, that is, the number of fully active ATPase sites per total sites, was estimated from the amplitude $(A)$ of the transient $\mathrm{Pi}$ burst. Experiments were performed in relaxed conditions because, first, $\mathrm{A}$ is similar in relaxed and in $\mathrm{Ca}^{2+}$-activated conditions, the equilibrium constant of the cleavage step that controls the Pi burst amplitude occurring in detached state whatever the condition. Second, A is 
determined more accurately in relaxed than in activated conditions, because the steady state is slower. Third, with myofibrils, A is similar to the amplitude of titration experiments (cold ATP chase), because the equilibrium constant of the cleavage step is large $(23,27)$.

Stopped-flow experiments. This type of experiment allows the measurements of the kinetics of ATP binding induces myosin head detachment from the thin filament and of the evaluation of the ATP cleavage step.

The experiment was carried out in a Hi-Tech Scientific stoppedflow apparatus (model SF-61 DX2, Hi-Tech Limited, Salisbury, UK). The excitation wavelength was $295 \mathrm{~nm}$, and emission was $>320 \mathrm{~nm}$ using a cut-off filter (WG320, Hi-Tech Limited, Salisbury, UK) to measure the tryptophan fluorescence. For each experimental condition, a series of 12 shots were carried out and averaged. The fluorescence of the myofibrillar solution without ATP, was measured just before the commencement of the experiment and was set as background. This latter was subtracted to the signal of the reaction mixture with ATP. For further details, see Stehle et al. (36).

Myosin isoform determination. Muscles to be used for electrophoresis were quickly frozen in liquid $\mathrm{N}_{2}$ after dissection, pulverized in a small steel mortar, and stored at $-80^{\circ} \mathrm{C}$ until analysis. Myofibrillar proteins were extracted from 7 to $10 \mathrm{mg}$ of dry muscle powder, as described previously (8), washed first with a solution containing 6.3 mM EDTA (pH 7), $0.1 \%$ pepstatin, $1 \%$ PMSF, and then with a second solution containing $50 \mathrm{mM} \mathrm{KCl}, 0.1 \%$ pepstatin, $1 \%$ PMSF. The myofibrillar proteins were resuspended in $1 \mathrm{ml}$ of sterile milliQ water and their concentration determined by a protein assay kit (Dc Protein Assay, Bio-Rad) to prepare samples with a final amount of $10 \mu \mathrm{g}$ for one-dimensional electrophoresis or $70 \mu \mathrm{g}$ for two-dimensional electrophoresis. Proteins were precipitated for $2 \mathrm{~h}$ with acetone $(8 \mathrm{v} / \mathrm{v})$, followed by centrifugation for $1 \mathrm{~h}$ at $13,000 \mathrm{rpm}$. The pellet was dissolved in Laemmli solution for SDS-PAGE or in rehydration buffer for two-dimensional gel electrophoresis. For MHC isoforms separation, $\sim 1.50-1.75 \mu \mathrm{g}$ proteins were loaded on each lane of an $8 \%$ polyacrylamide SDS gel (with a $4 \%$ stacking gel) and run for $24 \mathrm{~h}$ at $4^{\circ} \mathrm{C}$ with $180 \mathrm{~V}$ (constant voltage; current $\sim 13 \mathrm{~mA} / \mathrm{gel}$ ). Gels were stained using Coomassie blue techniques, and four bands were separated, corresponding to MHC-I, MHC-IIA, MHC-IIX, and MHC-IIB isoforms. For MLC isoform separation, 12\% separating gels with $4 \%$ stacking gels were used. The electrophoresis was run for $8 \mathrm{~h}$ at low temperature $(150 \mathrm{~V}, 13 \mathrm{~mA} / \mathrm{gel})$. Gels were stained with Coomassie blue and five isoforms, two regulatory-MLC2s and MLC2f-and three alkali-MLC1f, MLC1s, and MLC3f-were identified. Separation of variants of MLC isoforms on two-dimensional gel electrophoresis was achieved with a procedure similar to those previously reported (9). For the first dimension, isoelectric focusing, proteins were solubilized in a buffer containing $8 \mathrm{M}$ urea, 2\% CHAPS, $10 \mathrm{mM}$ DTT, and 2\% carrier ampholites (Amersham Biosciences, Piscataway, $\mathrm{NJ}$ ) and then separated with the Ettan IPGphor Isoelectric Focusing System (Amersham Biosciences) on 3.5\% acrylamide strips with immobilized $\mathrm{pH}$ gradients. Strips were rehydrated at $50 \mathrm{~V}$ for $12 \mathrm{~h}$, and proteins were focused under the following voltage conditions: 500 $\mathrm{V}$ for $1 \mathrm{~h}, 500-1,000 \mathrm{~V}$ for $1 \mathrm{~h}, 5,000 \mathrm{~V}$ until reaching $100,000 \mathrm{~V} / \mathrm{h}$. Temperature was kept constant at $26^{\circ} \mathrm{C}$. After reduction with a buffer containing $6 \mathrm{M}$ urea, 30\% glycerol, $375 \mathrm{mM}$ Tris $\cdot \mathrm{HCl}(\mathrm{pH} 8.8), 2 \%$ DTT, and alkylation with the same buffer with the addition of $2.5 \%$ iodoacetamide, the strips were embedded in $4 \%$ polyacrylamide stacking gels, and the proteins were separated in $12 \%$ polyacrylamide gels for $8 \mathrm{~h}$ at $150 \mathrm{~V}$ and low temperature $\left(4^{\circ} \mathrm{C}\right)$. Finally, electrophoresis gels were silver stained according to Oakley et al. (29).

For image analysis and quantification, the electrophoretic gels were digitized with an Epson 1650 scanner with 1,200 dpi resolution. The densitometric profile was obtained by measuring the area covered by each band of MHC or MLC isoforms and expressed as \% of the total area of the peaks corresponding to MHC, to alkali MLC, or to regulatory MLC, respectively. The spots of the two-dimensional gels were analyzed determining brightness-area product (BAP) with a constant threshold after black/white inversion with Adobe Photoshop software. In each gel, the BAP values of the spots identified as slow and fast MLC-2 were summed to give a total for slow MLC-2 and a total for fast MLC-2. The value of each spot identified as slow MLC-2 was expressed as a percentage of total slow MLC-2, and the same was done for the spots identified as fast MLC-2. The positions of slow and fast isoforms of MLC on two-dimensional gels were determined according to their molecular weight, using appropriate markers of molecular weight in the second dimension and to their isoelectric point in the first dimension. From percentage values obtained in different gels, means (SD) were calculated.

Statistical analysis. Training and hypoxia effects were analyzed by means of a two-way ANOVA after testing the conditions of application (normality of distributions and homogeneity of variances). The two factors were exercise activity (sedentary $v s$. training) and environment (normoxia vs. hypoxia). $P<0.05$ was taken as the level of statistical significance. In case of a significant main effect, a StudentNewman-Keuls post hoc test was applied (Statistica, Statsoft, Tulsa, $\mathrm{OK})$. Mean and standard deviation of the mean (SD) were presented.

\section{RESULTS}

Training adaptations and body weight. MAV increased $(P<0.05)$ from $37.6 \pm 3.1$ and $37.1 \pm 4.7 \mathrm{~m} / \mathrm{min}$ for NT and HT, respectively at the beginning of the protocol under normoxic conditions to $43.7 \pm 2.6$ and $44.0 \pm 3.7 \mathrm{~m} / \mathrm{min}$ for NT and HT, respectively, after 5 wk of training. Initially, there was no significant difference in body mass between groups. The rats' body weight increased significantly $(P<0.05)$ after the 5-wk protocol, but without any differences between groups: $\mathrm{N}$ : $243 \pm 8.0$ g vs. $258 \pm 23 \mathrm{~g}$; NT: $237 \pm 21$ g vs. $248 \pm 25 \mathrm{~g}$; H: $244 \pm 21 \mathrm{~g}$ vs. $258 \pm 21 \mathrm{~g}$; and HT: $244 \pm 11 \mathrm{~g}$ vs. $249 \pm$ $14 \mathrm{~g}$ before and after the protocol, respectively.

Sarcomere length. The sarcomere length of soleus myofibrils $(2.4 \mu \mathrm{m})$ was in accordance with the reported values in the literature. It was homogeneous among myofibrils of each group. The myofibrils were rectilinear and not aggregated.

$\mathrm{Ca}^{2+}$-activated ATPase activity. Myofibril ATPase activity was followed over $200 \mathrm{~s}$ and, in the presence of $\mathrm{Ca}^{2+}$, the three distinct phases mentioned above could be identified (Fig. 1): 1) a fast monoexponential phase on the milliseconds time scale, corresponding to a transient Pi burst phase of amplitude A (kinetics, $k_{\mathrm{obs}}$, not obtained on the timescale used), 2) a fast linear phase with a slope $k_{\mathrm{F}}$, corresponding to the myofibril ATPase activity during unloaded shortening, 3) a slow linear phase with a slope $k_{\mathrm{S}}$, corresponding to the myofibril activity during overcontraction. Because this last phase is not physiological, no further interest was given to $k_{\mathrm{S}}$. The transition between $k_{\mathrm{F}}$ and $k_{\mathrm{S}}$ occurs at time $t_{\mathrm{B}}$, which is inversely related to the unloaded shortening speed. The three characteristic phases were described with the triphasic model detailed in the methods section. The value of the kinetic parameters $k_{\mathrm{F}}$ and $t_{\mathrm{B}}$, essential for the comparison of ATPase activities, are given in Table 1. Three independent experiments using different myofibrillar preparations were performed, and the coefficient of variability for the three sets of the experiment was $7.8 \% \pm$ $1.6 \%$. This coefficient took into account experimental errors on myofibrils and ATP solution preparations and temperature fluctuations. Within each set of experiment, this kind of experimental error is likely to be lower, due to better control of external factors (same buffers, same day). 

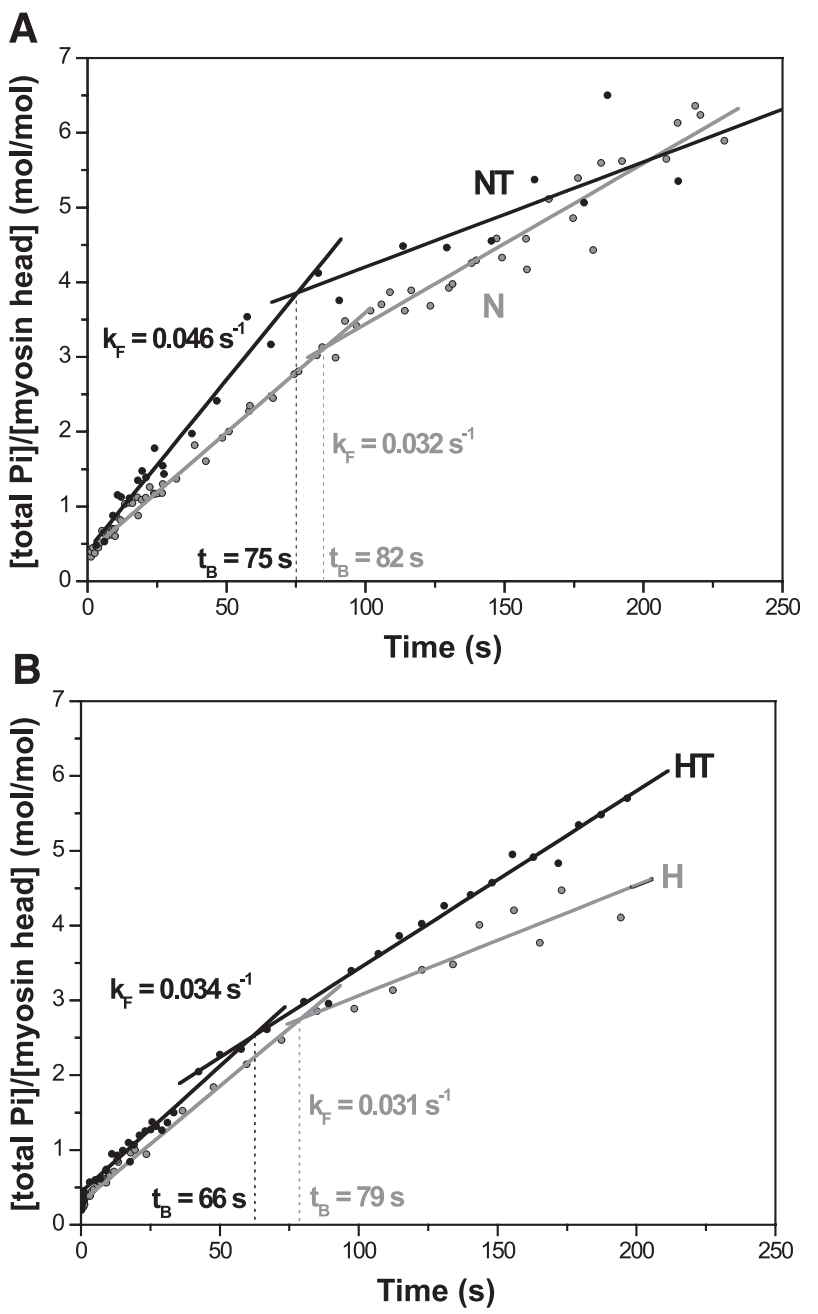

Fig. 1. Training effect on the soleus myofibrillar ATPase activity at $4^{\circ} \mathrm{C}$ in normoxia $(A)$ and hypoxia $(B)$ for the first series of separated experiments. The reaction mixture $\left(2.5 \mu \mathrm{M}\right.$ myosin heads $\left.+25 \mu \mathrm{M}\left[\gamma_{-}{ }^{32} \mathrm{P}\right] \mathrm{ATP}\right)$ was quenched in acid at the times indicated (between $200 \mathrm{~ms}$ and $250 \mathrm{~s}$ ), and the ${ }^{32} \mathrm{Pi}$ was determined. $\mathrm{k}_{\mathrm{F}}$ represents the slope of the fast linear phase; $\mathrm{tB}$ represents the duration of the unloaded shortening; $\mathrm{N}$, normoxic group $(n=14)$; NT, normoxic training group $(n=14) ; \mathrm{H}$, chronic hypoxic group $(n=10)$; HT, chronic hypoxic training group $(n=14)$.

Effects of training and/or hypoxia on $\mathrm{Ca}^{2+}$-activated myofibrillar ATPase activity. The ATPase activities were consistent among the three series of independent repetitions, and variance analysis showed a significant main effect of training as the slope, $k_{\mathrm{F}}$, was significantly higher $(P<0.01)$ in the training groups compared with the sedentary groups whatever the environment of exposure (normobaric normoxia or hypobaric hypoxia) (Table 1). Post hoc tests indicate a difference between N and NT groups. In the same line, the duration of the unloading shortening phase, $t_{\mathrm{B}}$, was shorter (main effect $P<$ $0.05)$ in training groups compared with sedentary groups. Because shortening velocity is a function of $t_{\mathrm{B}}$, this decrease suggests an increase in the unloading shortening velocity with training. As far as hypoxia, no significant main effect was detected, and only a trend $(P=0.07)$ for a decrease in $k_{\mathrm{F}}$ was observed in hypoxic groups, whatever the training status.

Titration of active myosin heads. The estimated values of $A$ were similar in the NT and N groups $(0.48$ and $0.47 \mathrm{~mol} / \mathrm{mol}$, respectively) suggesting that the positive effect of training on the myofibrillar ATPase activity is not due to an increase in the number of fully competent myosin heads (Fig. 2). As far as the effect of hypoxia, the values of Pi burst amplitude were similar in the $\mathrm{H}$ and control groups $(0.49$ and $0.44 \mathrm{~mol} / \mathrm{mol}$, respectively) suggesting that moderate hypoxia had no effect on the number of fully competent myosin heads.

Cleavage step rate. The rate of the ATP cleavage step was evaluated from tryptophan fluorescence stopped-flow experiments with relaxed myofibrils, as previously described. The slow-phase rate constant had similar values in $\mathrm{N}(8.5 \pm 1.2$ $\left.\mathrm{s}^{-1}\right)$ and in NT groups $\left(9.7 \pm 2.7 \mathrm{~s}^{-1}\right)$, suggesting that training did not affect the cleavage step kinetics. The cleavage step rates were evaluated only in the normoxic groups because the hypoxic effect on m-ATPase did not reach significance level.

$M H C$ and $M L C$ isoform identification and quantification. Table 2 shows the distribution of slow and fast MHC and MLC isoforms of soleus, determined by one-dimensional gel electrophoresis. Examples of MHC and MLC separation performed with precisely the same protocol can be seen in Fig. 1 in Bozzo et al. (9). In hypoxic conditions, the proportion of the fast isoforms was increased $(P<0.01$ and $P<0.05$ for MHC-IIX and MHC-IIB, respectively), and the proportion of slow isoforms was decreased $(P<0.01)$ compared with the normoxic control and training groups. Thus, a slow-to-fast transition was induced by hypoxia. Training led to an increase in MHC-I $(P<0.01)$, indicating an opposite effect to hypoxia. Training in hypoxic conditions caused a reduction of the slow-to-fast transition induced by hypoxia alone, as indicated by the decreased proportion of MHC-2A. Training effect was less pronounced than the hypoxic one.

MLC isoform expression showed similar changes to those observed in MHC isoforms. Hypoxia produced an increase in fast isoforms $(P<0.001$ for both MLC-1f +3 and MLC-2f $)$ and a decrease in slow isoforms $(P<0.001$ for both MLC-1s and MLC-2s). These changes were partially reversed by training in hypoxic conditions. Again, the training effect was less pronounced than hypoxic effect, and its effect was detectable only for MLC-1. MLC-1s increase and MLC-1f+3 decrease $(P<0.01)$.

Two-dimensional gel electrophoresis resolved the regulatory MLC isoforms in several spots, corresponding to variants with

Table 1. Average soleus myofibrillar ATPase activity $\left(k_{F}\right)$ and duration of the shortening phase $\left(t_{B}\right)$ in activated condition at $4^{\circ} \mathrm{C}$ in the four experimental groups

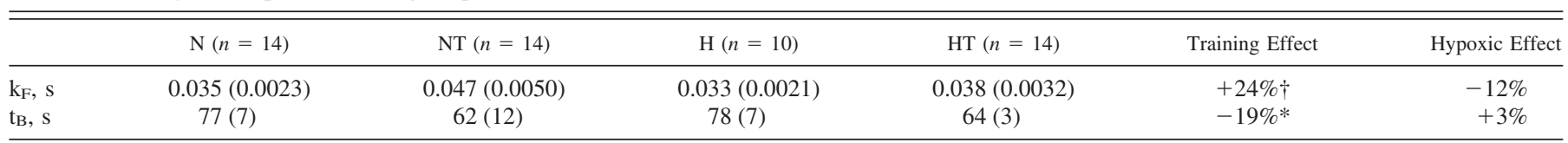

Values are expressed as means (SD). N, normoxic group; NT, normoxic training group; H, chronic hypoxic group; HT, chronic hypoxic training group; $\mathrm{k}_{\mathrm{F}}$, the slope of the fast linear phase; $\mathrm{t}_{\mathrm{B}}$, duration of the shortening; $* P<0.05 ; \dagger P<0.01 ; n=$ number of muscles. 


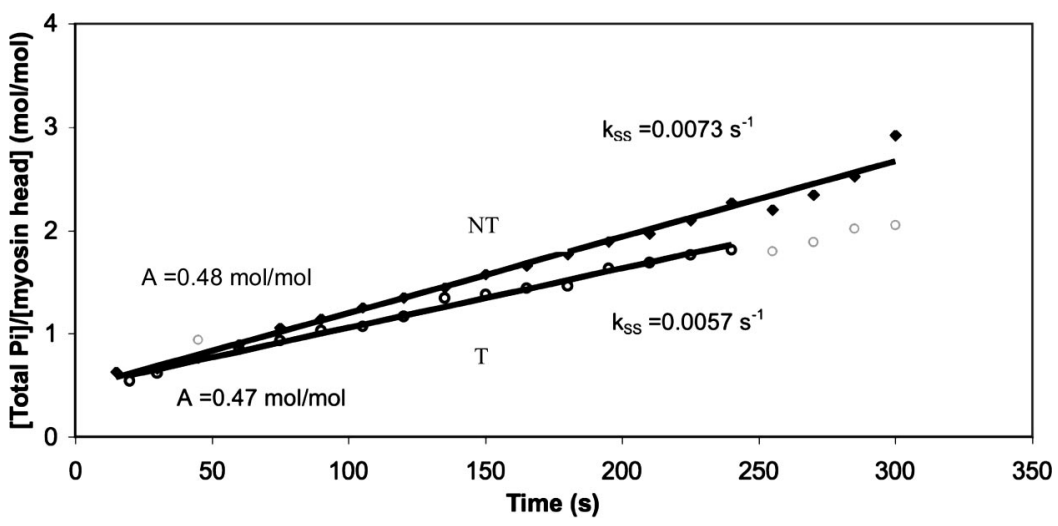

Fig. 2. Amplitude of the Pi burst and evaluation of myosin ATPase titration in normoxic group $(\mathrm{N} ; n=14)$ and normoxic training group (NT; $n=14$ ). The reaction mixture was $2 \mu \mathrm{M}$ myosin heads and $10 \mu \mathrm{M}\left[\gamma^{-32} \mathrm{P}\right] \mathrm{ATP}$ in relaxed condition. No significant effect of training could be evidenced in the amplitude of the Pi burst.

distinct isoelectric points [an example is given in Fig. 2 in Bozzo et al. (9)]. In the soleus, slow MLC-2 was the predominant regulatory light chain and appeared separated into two spots (s and s1), with different isoelectric points. A third smaller spot was also present and corresponded to a variant MLC-2f, whereas the acidic variant MLC-f1 was undetectable. The average proportions of the MLC-2 variants are shown in Table 3. No significant changes in the distribution between less acidic nonphosphorylated variants ( $\mathrm{s}$ and $\mathrm{f}$ ) and more acidic phosphorylated variants (s1 and $\mathrm{f} 1$, which was undetectable) were present in the soleus of the four experimental groups.

\section{DISCUSSION}

The aim of the present study was to determine whether endurance training and/or hypoxia caused different changes in myofibrillar ATPase activity in relation with the known changes in MHC isoform composition in rat soleus. Myofibrils were prepared from the soleus muscle of rats trained in normoxic or hypoxic conditions for $5 \mathrm{wk}$. The determination of myofibrillar ATPase activity and MHC isoform composition of soleus myofibrils showed that 1 ) endurance training increased significantly the myofibrillar ATPase activity, although a fastto-slow shift in MHC isoform distribution took place; 2) in spite of a slow-to fast transition in MHC isoform distribution observed, no concomitant increase was evident in hypoxic groups and in contrast chronic hypoxia tended $(P=0.07)$ to decrease the myofibrillar ATPase activity. It is generally accepted that MHC isoforms are the major determinants of myofibrillar ATPase activity. The issue has been investigated on many preparations from isolated myosin to single muscle fibers. The contribution of the present study points out a possible modulation of the relation between MHC isoforms and ATPase activity. Actually, in the present experimental conditions, ATPase activity and MHC isoforms appeared dissociated, and other molecular control mechanisms were evoked.

Potential limits. One could argue that the observed changes in ATPase activity were temperature dependent. However, we examined previously the three characteristic phases of myofibril ATPase kinetics in unloading myofibrils under a large range of temperature $\left(4\right.$ to $\left.35^{\circ} \mathrm{C}\right)$, and the Arrhenius plots of all the kinetics constants, including the duration of shortening phase were linear, indicating that the energy of activation was constant for all temperatures studied; that is, no break in the Arrhenius plot has been observed (11). Therefore, one may argue that the same limiting steps are controlling the ATPase activity over this large range of temperature. At low temperatures, myofibrils displayed the same behavior than at high temperatures, and all the contractile characteristics (including the shortening phase) were conserved, the only difference being the rate of the reaction. Whatever the temperatures, $\sim 3$ ATP are consumed during the shortening phase. The chosen temperature of $4^{\circ} \mathrm{C}$ although slowing down the shortening velocity, still allows the myofibrils to behave and contract normally. Interestingly, myofibrils prevented from shortening with 1-ethyl3-[3-dimethylaminopropyl]carbodimide hydrochloride). cross-linker ( $8 \%$ of the myosin head cross-linked) displayed a different kinetics than native myofibrils. Only one linear phase corresponding to an isometric action occurred, whereas in native myofibrils, two linear phases with different slopes were evi-

Table 2. Distribution of MHC and MLC isoforms of soleus determined by one-dimensional gel electrophoresis

\begin{tabular}{|c|c|c|c|c|c|c|c|}
\hline & $\mathrm{N}(n=14)$ & $\mathrm{H}(n=10)$ & NT $(n=11)$ & $\mathrm{HT}(n=13)$ & Training Effect & Hypoxia Effect & Interaction \\
\hline MHC-IIA & $25.8(5.2)$ & $34.5(12.1)$ & $25.4(7.7)$ & $24.2(9.7)$ & & & \\
\hline MHC-IIX & $1.0(1.9)$ & $5.9(7.4)$ & $0.4(1.2)$ & $2.0(2.9)$ & & $\dagger$ & \\
\hline MLC-1 s & $81.1(7.4)$ & $70.4(4.2)$ & $81.8(3.2)$ & $78.1(3.2)$ & $\dagger$ & $\ddagger$ & * \\
\hline MLC- $1 \mathrm{f}+3$ & $18.8(7.4)$ & $29.4(4.2)$ & $18.2(3.2)$ & $21.9(3.2)$ & $\dagger$ & $\ddagger$ & * \\
\hline MLC-2 s & $77.3(6.2)$ & $54.4(4.2)$ & $72.1(3.3)$ & $64.3(2.1)$ & & $\ddagger$ & \$ \\
\hline
\end{tabular}

Values are expressed as means (SD). Number $(n)$ of muscles examined is in parentheses. MHC I, slow-type myosin heavy chain; MHC IIA, intermediate-type myosin heavy chain; MHC IIX, fast intermediate-type myosin heavy chain; MHC IIB, fast-type myosin heavy chain; MLC $1 \mathrm{~s}$, slow myosin light chain type 1; MLC 1f, regulatory fast myosin light chain type 1; MLC 3, fast myosin light-chain type 3; MLC $2 \mathrm{~s}$, regulatory slow myosin light chain type 2; MLC 2f, fast myosin light chain type $2 . * P<0.05 ; \dagger P<0.01 ; \ddagger P<0.001$. 
Table 3. Distribution of slow and fast MLC-2 isoforms of soleus determined by two-dimensional gel electrophoresis

\begin{tabular}{rrrr}
\hline \hline $\mathrm{N}(n=5)$ & $\mathrm{NT}(n=4)$ & $\mathrm{H}(n=4)$ & $\mathrm{HT}(n=4)$ \\
\hline $73.9(2.4)$ & $71.9(2.0)$ & $73.1(2.0)$ & $72.0(2.0)$ \\
$26.1(2.3)$ & $28.1(2.0)$ & $26.9(1.9)$ & $28.0(1.9)$ \\
$100.0(0.0)$ & $100.0(0.0)$ & $100.0(0.0)$ & $100.0(0.0)$ \\
$0.0(0.0)$ & $0.0(0.0)$ & $0.0(0.0)$ & $0.0(0.0)$ \\
\hline
\end{tabular}

Values are expressed as means (SD). Number $(n)$ of samples analyzed is indicated in parentheses.

denced corresponding to shortening in unloading reaction and in pseudo-isometric condition. At $4^{\circ} \mathrm{C}$ with native myofibrils, the two linear phases were clearly observed (e.g., Fig. 1). Obviously, low temperatures offer a better time resolution and allow the cleavage step evaluation from tryptophan fluorescence stopped-flow experiments, similarly to Stehle et al. (36) at $4^{\circ} \mathrm{C}$.

Effects of endurance training on MHC isoform content. The effects of endurance training on muscle $\mathrm{MHC}$ isoforms are well established. Many investigations $(6,15,17,20,25,37,38$, 39) have demonstrated that this type of muscular activity promotes significant transformation of fast-to-slow MHC isoforms within both slow and fast skeletal muscles. Such shift in the total muscle MHC pool is not due to selective changes in fiber size but is due to a training-induced transformation in MHC isoforms expressed by individual fibers. In the present study, we observed also this significant phenotype shift from fast to slow type (Table 2). In contrast, Bigard et al. (6) did not detect any training effect on MHC content in EDL muscle. This might be explained by the high proportion of fast-twitch fibers in EDL and its pattern of activation during the recovery phase of running (i.e., no load).

Hypoxic effects on MHC isoform content. Several studies found a shift toward more glycolytic-oxidative fibers (MHCIIA) in rat muscles exposed to hypoxia, compared with control rats. Bigard et al. (7) showed that $4 \mathrm{wk}$ in hypobaric hypoxia resulted in a decrease in MHC-I in the soleus of rats and an increase in the fast-type isoforms. These authors studied the effects of endurance training in hypoxic conditions, that is, the combination of training and hypoxia, and found that endurance training re-established the MHC isoform profile similar to that observed in normoxia. Indeed, sedentary rats in chronic hypobaric hypoxia (4 wk) showed a significant decrease in type I MHC in soleus (12\%). Although strongly decreased under hypoxia, spontaneous running activity increased the expression of type I MHC so that no difference in the MHC profile of soleus was found between chronic hypoxic active and normoxic active rats. Thus, hypoxia seems to affect the structural and biochemical properties of skeletal muscle by inducing a transformation from type I to type II fibers. In the same line, in the present study, a significant slow-to-fast transition was observed in hypoxic conditions. Only Sillau and Banchero (35) found no effect of a hypoxic exposure on the MHC composition in rat soleus.

Endurance training effects on myofibrillar ATPase activity. The observed changes in ATPase activity under training effect in the soleus are in agreement with the previous finding of Schluter and Fitts (34). A 22\% increase in ATPase activity (similar to the $24 \%$ increase of the present study) was reported at the end of an 8-12 wk endurance training protocol. The increase in ATPase activity during endurance training seems to be specific for the slow fibers, which are likely more recruited during endurance exercise. The ATPase activity of the fast fibers, decreased after training or remained unchanged (34). In the present study, the m-ATPase activity increased independently of the transitions of MHC-II to MHC-I during training. The notion that adaptation in the properties of myosin molecule itself could take place is in agreement with previous studies. Chronic exercise was found to determine greater peak powergenerating capacity in skinned myocytes from chronically exercise-trained pigs compared with sedentary counterparts (21), although no MHC change was found in this study. In addition, the sliding velocity of skeletal muscle myosin 1 of a group of well-trained bodybuilders was different than that of myosin 1 of controls (14). In addition to training effect, age seems also to determine a change in the properties of the myosin molecule without a necessary change in myosin isoforms (13). In agreement with the well-established correlation between ATPase activity and maximal shortening velocity [e.g., Barany (3)], the positive effect of training on unloading shortening velocity is evidenced by the shorter duration of unloading shortening phase in our training groups. Changes in shortening velocity induced by training have been also reported by Schluter and Fitts (34) in soleus fibers with the same magnitude to those of myosin ATPase activity. In addition, Canepari et al. (12) reported an increase in the sliding velocity of actin filaments on myosin isoform IIa in human vastus lateralis muscle, following a 12-wk resistance training, whereas no significant increase was observed in the sliding velocity on myosin isoform I. The adaptive mechanisms seem to occur quite logically in the recruited fibers: intermediate type with progressive resistance training and slow type with endurance training. Of importance regarding the adaptation to endurance exercise: the slightly increased power and speed of the slow type I fibers makes them more suited for the rapid phasic patterns of exercise, whereas the slow tonic recruitment is associated with the main antigravity role of the slow fibers for sedentary.

Hypoxia effect on myofibrillar ATPase activity. As stated in the introduction, several studies have shown that chronic exposure to hypoxia induces a decrease in the relative content of MHC-I in all muscles, but especially in slow muscles as soleus. Thus, on the basis of the slow-to-fast transition in myosin isoform expression, an increase of ATPase activity would be expected following exposure to hypoxia. In this study for the first time, myofibrillar ATPase was determined in muscles exposed for many weeks to hypoxia corresponding to the altitude of $2,800 \mathrm{~m}$ and, contrary to expectations, ATPase activity was not increased in response to hypoxic stimulus. Indeed, a trend for a decrease was observed $(P=0.07)$ in hypoxia, compared with normoxic groups. Similar dissociations between MHC and functional adaptation to hypoxia were previously reported. After short-term intermittent hypoxia, fibers of sternohyoid muscle, an upper airway dilator, displayed a transition from MHC type $2 \mathrm{~A} / 2 \mathrm{~B}$ to MHC type $2 \mathrm{~B}$, whereas the muscle showed similar tension-frequency relationships compared with the control condition (30). After chronic hypoxia exposure, a significant decrease in myofibrillar ATPase activity was reported in the right ventricle of sheep, although no change in myosin isoform occurred (24). In addition, slight 
and significant depressive effects of hypoxia on $\mathrm{Na}^{+}-\mathrm{K}^{+}$. ATPase activity were reported $(1,18,19)$, suggesting an occurrence of an adaptive mechanism also present in ATPase pump activity.

Molecular mechanisms of ATPase activity. Most studies on the relationship between MHC isoforms and kinetic parameters as $\mathrm{V}_{\max }$ or ATPase activity have compared muscles or single fibers with distinct MHC isoform composition. Few studies have analyzed whether kinetic parameters and MHC isoforms change concomitantly in the same muscle as an adaptation to new functional demands, such as endurance training or hypoxia. The present results show a dissociation, which implies that other factors, beside MHC isoforms, may modulate kinetic parameters. The first possible explanation lies in an increase in the number of myosin heads fully competent from an enzymatic point of view. Thus, the endurance training effect on myosin ATPase activity might be caused by a simple increase in the number of active heads. The assessment of titration of the active myosin heads based on Pi burst amplitude, however, suggests that training did not modify the number of fully competent myosin heads. In addition, the experimental approach adopted in this study allowed us to analyze, at least in part, the individual steps of the ATPase cycle. Because the steady-state rate is mainly controlled by the slowest step rates (rate-limiting steps), the increase of the ATPase activity caused by endurance training may be attributed to an increase in the rate of such rate-limiting steps. The steady state depends on the isomerization step that controls Pi release, $k_{4}$, in the kinetics scheme of Bagshaw and Trentham (2) and the cleavage step equilibrium constant $\left(K_{3}\right)$ by the following equation: $k_{\mathrm{cat}}=k_{4}$ $K_{3} /\left(1+K_{3}\right)$, where $k_{\text {cat }}=k_{\mathrm{F}} /$ [active site], $k_{4}$ is the rate constant of the isomerization step preceding Pi release, $K_{3}$ is the cleavage step equilibrium constant, $K_{3}=k_{+3} / k_{-3}$ with $\mathrm{k}_{+3}$ and $\mathrm{k}_{-3}$ the forward and backward cleavage step kinetics rate (e.g., 23). In soleus as in psoas myofibrils during unloaded shortening, the kinetics of production of total and free $\mathrm{Pi}$ indicate that the rate-limiting step is clearly an isomerization step preceding, and thus controlling, the $\mathrm{Pi}$ release. This conclusion is mainly based on the occurrence of a large total Pi burst and the lack of free Pi burst. Because the ATP cleavage occurs in an actin-detached configuration of myosin, its kinetics can be indifferently studied in activated or relaxed conditions. In this study, ATP cleavage was evaluated by means of the slow phase of tryptophan fluorescence signal upon mixing relaxed myofibrils with ATP in a stopped-flow apparatus, as proposed by Stehle et al. (36). No training effect could be detected on the ATP cleavage kinetics. This suggests that the training effect on the steady-state ATPase is due to an increase in the slowest step, the rate of the isomerization step preceding Pi release. Several studies $(18,33,38)$ have proposed that regulatory myosin light chains (MLC-2) may play a role in the adaptations following training. These studies showed that the proportion of fast regulatory MLC (MLC-2f) is increased by endurance training in the slow muscles, and this would induce an increase in ATPase activity. After a 10-wk endurance progressive running program, the MLC-3f decreased by $61 \%$ $(P<0.01)(38)$. These authors suggested that this 10 -wk progressive running program decreased the contractile velocity of type IIB fibers and that the observed training-induced decrease of the muscle contractile velocity might be due to changes in fast alkali MLC complements within a certain fiber type but also due to shifts in MHC-based fiber populations. In addition, Green et al. (18) demonstrated that a prolonged (15 wk) endurance training induced a fiber transformation from fast type IIA to slow type I on the basis of enzyme histochemistry and MLC distribution. We hypothesize that the changes in ATPase activity and shortening duration might be attributable to an altered expression of fast MLC-2 in the slow type I fibers. This hypothesis is supported by the fact that light chains are known to be involved in the power stroke and that removal of light chains depresses force and velocity. However, in the present study, no significant change in MLC-2 isoforms between the sedentary $(\mathrm{N}$ and $\mathrm{H})$ and the trained groups (NT and HT) were detected. The lack of changes in MHC II and MLC-2 isoform distribution under training effect might be explained, as suggested by Salmons (33), assuming that a minimal duration and intensity of exercise is required to change in myosin subunits. Regulatory MLC can be phosphorylated and when MLC-2 is phosphorylated, force developed at submaximal calcium concentration is increased, that is, myofibrils become more sensitive to calcium activation. In the present study the level of MLC-2 phosphorylation was determined with twodimensional gel electrophoresis, and no significant change in the MLC-2 phosphorylation was detected between sedentary and trained groups. Thus, MLC-2 phosphorylation cannot explain the increase in ATPase activity. Attempts to explain the lack of relationship between myofibrillar ATPase activity changes and the changes in the distribution of MLC isoforms and of their level of phosphorylation were not successful. In the same way as for the increase of myofibrillar ATPase due to endurance training, it is more likely that the enzymatic activity is influenced by other myofibrillar proteins different from myosin or by posttranslational modification of myosin, different from MLC-2 phosphorylation. The identification of such a myofibrillar protein or of the relevant posttranslational modifications of myosin is beyond the scope of the present study.

Conclusion. Although it is generally accepted that myofibrillar ATPase activity is controlled by MHC isoforms, the present study showed that under specific circumstances, dissociations might occur. In this study, the analysis of MHC isoform transition and myofibrillar ATPase changes after endurance training gave a first example that the quantitative variations in the myofibrillar ATP hydrolysis rate are a result of complex mechanisms depending undoubtedly of the myosin isoforms but also under the control of other less known factors. The increased myosin ATPase activity in response to training was not linked to any effect on the hydrolysis step rate, suggesting that the slowest step of the mechano-chemical cross-bridge cycle, that is, the isomerization step preceding $\mathrm{Pi}$ release, is involved in this adaptation. A second example was found in chronic hypoxia in which in spite of the shift of MHC isoforms from slow to fast type, no significant change in ATPase activity was evidenced. Changes in expression of MLC isoform and in the level of MLC phosphorylation could not explain the dissociation between myofibrillar ATPase activity and MHC isoforms. Therefore, changes in other sarcomeric proteins or posttranslational modifications of myosin are likely to be involved.

\section{ACKNOWLEDGMENTS}

This work was partially supported by European Union Grant HPRN-CT2000-0091 to B. Roels and Italian Ministry of University through PRIN 
(Research Project of National Interest) Grant 2004 and the Royal Society Short Visit Grant.

\section{REFERENCES}

1. Aughey RJ, Clark SA, Gore CJ, Townsend NE, Hahn AG, Kinsman TA, Goodman C, Chow CM, Martin DT, Hawley JA, McKenna MJ. Interspersed normoxia during live high, train low interventions reverses an early reduction in muscle $\mathrm{Na}^{+}, \mathrm{K}^{+}$ATPase activity in well-trained athletes. Eur J Appl Physiol 98: 299-309, 2006.

2. Bagshaw CR, Trentham DR. Transient kinetic and isotopic tracer studies of the myosin adenosine triphosphatase reaction. J Supramol Struct 3: 315-322, 1975.

3. Barany M. ATPase activity of myosin correlated with speed of muscle shortening. J Gen Physiol 50 Suppl:197-218, 1967.

4. Barman TE, Travers F. The rapid-flow-quench method in the study of fast reactions in biochemistry: extension to subzero conditions. Methods Biochem Anal 1-59, 1985.

5. Bigard AX, Brunet A, Guezennec CY, Monod H. Skeletal muscle changes afterendurance training at high altitude. J Appl Physiol 2114 2121, 1991.

6. Bigard XA, Janmot C, Merino D, Lienhard F, Guezennec YC, D'Albis A. Endurance training affects myosin heavy chain phenotype in regenerating fast-twitch muscle. J Appl Physiol 81: 2658-2665, 1996.

7. Bigard AX, Sanchez H, Birot O, Serrurier B. Myosin heavy chain composition of skeletal muscles in young rats growing under hypobaric hypoxia conditions. J Appl Physiol 88: 479-486, 2000.

8. Bozzo C, Stevens L, Bouet V, Montel V, Picquet F, Falempin M, Lacour M, Mounier Y. Hypergravity from conception to adult stage: effects on contractile properties and skeletal muscle phenotype. J Exp Biol 2793-2802, 2004.

9. Bozzo C, Spolaore B, Toniolo L, Stevens L, Bastide B, CieniewskiBernard C, Fontana A, Mounier Y, Reggiani C. Nerve influence on myosin light chain phosphorylation in slow and fast skeletal muscles. FEBS J: 272: 5771-5785, 2005.

10. Burtscher M, Nachbauer W, Baumgartl P, Philadelphy M. Benefits of training at moderate altitude versus sea level training in amateur runners. Eur J Appl Physiol Occup Physiol 74: 558-563, 1996.

11. Candau R, Iorga B, Travers F, Barman T, Lionne C. At physiological temperatures the ATPase rates of shortening soleus and psoas myofibrils are similar. Biophys J 3132-3141, 2003.

12. Canepari M, Rossi R, Pellegrino MA, Orrell RW, Cobbold M, Harridge S, Bottinelli R. The effects of resistance-training on myosin function studied using the in vitro motility assay in young and older men. J Appl Physiol 98: 2390-2395, 2005.

13. D'Antona G, Pellegrino MA, Adami R, Rossi R, Carlizzi CN, Canepari M, Saltin B, Bottinelli R. The effect of ageing and immobilization on structure and function of human skeletal muscle fibres. J Physiol 15: 499-511, 2003.

14. D'Antona G, Lanfranconi F, Pellegrino MA, Brocca L, Adami R, Rossi R, Moro G, Miotti D, Canepari M, Bottinelli R. Skeletal muscle hypertrophy and structure and function of skeletal muscle fibres in male body builders. $J$ Physiol 570: 611-627, 2006.

15. Fitzsimons DP, Diffee GM, Herrick RE, Baldwin KM. Effects of endurance exercise on isomyosin patterns in fast- and slow-twitch skeletal muscles. J Appl Physiol 68:1950-1955, 1990.

16. Goret L, Reboul C, Tanguy S, Dauzat M, Obert P. Training does not affect the alteration in pulmonary artery vasoreactivity in pulmonary hypertensive rats. Eur J Pharmacol 527: 121-128, 2005.

17. Green HJ, Klug GA, Reichmann H, Seedorf U, Wiehrer W, Pette D. Exercise-induced fibre type transitions with regard to myosin, parvalbumin, and sarcoplasmic reticulum in muscles of the rat. Pflügers Arch 432-438, 1984.

18. Green H, MacDougall J, Tarnopolsky M, Melissa NL. Downregulation of $\mathrm{Na}^{+}-\mathrm{K}^{+}$- ATPase pumps in skeletal muscle with training in normobaric hypoxia. J Appl Physiol 86: 1745-1748, 1999.
19. Green H, Roy B, Grant S, Tupling R, Otto C, Pipe A, McKenzie D, Ouyang J. Effects of a 21-day expedition to 6,194 m on human skeletal muscle SR Ca ${ }^{2+}$-ATPase. High Alt Med Biol 1: 301-310, 2000.

20. Gregory P, Low RB, Stirewalt WS. Changes in skeletal-muscle myosin isoenzymes with hypertrophy and exercise. Biochem J 238: 55-63, 1986. Erratum in: Biochem J 240: following 933, 1986.

21. Hinken AC, McDonald KS. Beta-myosin heavy chain myocytes are more resistant to changes in power output induced by ischemic conditions. Am J Physiol Heart Circ Physiol 290: H869-H877, 2006.

22. Hoppeler H, Vogt M. Muscle tissue adaptations to hypoxia. J Exp Biol 204: 3133-3139, 2001.

23. Iorga B, Candau R, Travers F, Barman T, Lionne C. Does phosphate release limit the ATPases of soleus myofibrils? Evidence that (A)M. ADP. $\mathrm{P}(\mathrm{i})$ states predominate on the cross-bridge cycle. J Muscle Res Cell Motil 367-378, 2004.

24. Kamitomo M, Onishi J, Gutierrez I, Stiffel VM, Gilbert RD. Effects of long-term hypoxia and development on cardiac contractile proteins in fetal and adult sheep. J Soc Gynecol Investig 9: 335-341, 2002.

25. Kirschbaum BJ, Heilig A, Hartner KT, Pette D. Electrostimulationinduced fast-to slow transitions of myosin light and heavy chains in rabbit fast-twitch muscle at the mRNA level. FEBS Lett 243: 123-126, 1989.

26. Lionne C, Travers F, Barman T. Mechanochemical coupling in muscle: attempts to measure simultaneously shortening and ATPase rates in myofibrils. Biophys $J$ 887-895, 1996.

27. Lionne C, Iorga B, Candau R, Travers $\mathbf{F}$. Why choose myofibrils to study muscle myosin ATPase? J Muscle Res Cell Motil 24: 139-48, 2003.

28. Melin A, Fauchier L, Dubuis E, Obert P, Bonnet P. Heart rate variability in rats acclimatized to high altitude. High Alt Med Biol 4: 375-387, 2003.

29. Oakley BR, Kirsch DR, Morris NR. A simplified ultrasensitive silver stain for detecting proteins in polyacrylamide gels. Anal Biochem 361363, 1980.

30. Pae EK, Wu J, Nguyen D, Monti R, Harper RM. Geniohyoid muscle properties and myosin heavy chain composition are altered after shortterm intermittent hypoxic exposure. J Appl Physiol 98: 889-894, 2005.

31. Reboul C, Tanguy S, Gibault A, Dauzat M, Obert P. Chronic hypoxia exposure depresses aortic endothelium-dependent vasorelaxation in both sedentary and trained rats: involvement of L-arginine. J Appl Physiol 99: 1029-1035, 2005.

32. Reimann EM, Umfleet RA. Selective precipitation of ${ }^{32} \mathrm{Pi}$ onto filter papers. Application to ATPase and cyclic AMP phosphodiesterase determination. Biochim Biophys Acta 523: 516-521, 1978.

33. Salmons S. Exercise, stimulation and type transformation of skeletal muscle. Int J Sports Med 15: 136-141, 1994.

34. Schluter JM, Fitts RH. Shortening velocity and ATPase activity of rat skeletal muscle fibers: effects of endurance exercise training. Am J Physiol Cell Physiol 266: C1699-C1713, 1994.

35. Sillau AH, Banchero N. Effects of hypoxia on capillary density and fiber composition in rat skeletal muscle. Pflügers Arch 370: 227-232, 1977.

36. Stehle R, Lionne C, Travers F, Barman T. Kinetics of the initial steps of rabbit psoas myofibrillar ATPases studied by tryptophan and pyrene fluorescence stopped-flow and rapid flow-quench. Evidence that crossbridge detachment is slower than ATP binding. Biochemistry 39: $7508-$ $7520,2000$.

37. Sullivan VK, Powers SK, Criswell DS, Tumer N, Larochelle JS, Lowenthal D. Myosin heavy chain composition in young and old rat skeletal muscle: effects of endurance exercise. J Appl Physiol 78: 21152120, 1995.

38. Wada M, Inashima S, Yamada T, Matsunaga S. Endurance traininginduced changes in alkali light-chain patterns in type IIB fibers of the rat. J Appl Physiol 94: 923-929, 2003.

39. Wahrmann JP, Winand R, Rieu M. Plasticity of skeletal myosin in endurance trained rats (I). A quantitative study. Eur J Appl Physiol 84: 367-372, 2001. 